\title{
Clinical evidence and mechanistic basis for vildagliptin's effect in combination with insulin
}

This article was published in the following Dove Press journal:

Vascular Health and Risk Management

14 February 2013

Number of times this article has been viewed

\section{Anja Schweizer' \\ James E Foley ${ }^{2}$ \\ Wolfgang Kothny ${ }^{2}$ \\ Bo Ahrén ${ }^{3}$}

'Novartis Pharma AG, Basel, Switzerland; ${ }^{2}$ Novartis

Pharmaceuticals Corporation,

East Hanover, NJ, USA; ${ }^{3}$ Department of Clinical Sciences, Lund University, Lund, Sweden
Correspondence: Anja Schweizer

Novartis Pharma AG, Postfach, $\mathrm{CH}-4002$

Basel, Switzerland

$\mathrm{Tel}+4$ I 6I 3243530

Fax +4I 616963542

Email anja.schweizer@novartis.com
Abstract: Due to the progressive nature of type 2 diabetes, many patients need insulin as add-on to oral antidiabetic drugs (OADs) in order to maintain adequate glycemic control. Insulin therapy primarily targets elevated fasting glycemia but is less effective to reduce postprandial hyperglycemia. In addition, the risk of hypoglycemia limits its effectiveness and there is a concern of weight gain. These drawbacks may be overcome by combining insulin with incretinbased therapies as these increase glucose sensitivity of both the $\alpha$ - and $\beta$-cells, resulting in improved postprandial glycemia without the hypoglycemia and weight gain associated with increasing the dose of insulin. The dipeptidyl peptidase-IV (DPP-4) inhibitor vildagliptin has also been shown to protect from hypoglycemia by enhancing glucagon counterregulation. The effectiveness of combining vildagliptin with insulin was demonstrated in three different studies in which vildagliptin decreased A1C levels when added to insulin therapy without increasing hypoglycemia. This was established with and without concomitant metformin therapy. Furthermore, the effectiveness of vildagliptin appears to be greater when insulin is used as a basal regimen as opposed to being used to reduce postprandial hyperglycemia, since improvement in insulin secretion likely plays a minor role when relatively high doses of insulin are administered before meals. This article reviews the clinical experience with the combination of vildagliptin and insulin and discusses the mechanistic basis for the beneficial effects of the combination. The data support the use of vildagliptin in combination with insulin in general and, in line with emerging clinical practice, suggest that treating patients with vildagliptin, metformin, and basal insulin could be an attractive therapeutic option.

Keywords: DPP-4, GLP-1, GIP, incretin, insulin therapy, type 2 diabetes, hypoglycemia

When the findings from the Diabetes Control and Complications Trial and the United Kingdom Prospective Diabetes Study (UKPDS) were reported in the 1990s, the importance of good glycemic control became universally recognized. ${ }^{1-4}$ Further, the UKPDS established metformin as the "gold standard" first line monotherapy in the treatment of type 2 diabetes (T2DM) in most countries. With disease progression, a second and third oral agent were added; until recently these were usually a sulfonylurea (SU) or a thiazolidinedione (TZD). Short- or intermediate-acting insulin added before two to three meals each day was used only as a last resort when this triple oral therapy failed. Usually metformin and/or SU were maintained after initiation of insulin therapy, whereas TZDs were usually discontinued in patients without severe insulin resistance to avoid exacerbating the edema seen in some patients with insulin therapy.

All three of the aforementioned oral antidiabetic drugs (OADs) act to lower the basal glucose levels. Short-acting insulin provides a bolus action to lower glucose levels during meals, and the intermediate-acting insulin also includes a basal effect 
throughout the daytime. Although insulin-dosing regimens to control basal glucose during the night were utilized by specialists, this strategy was not widely employed.

More recently long-acting basal insulin, glucagonlike peptide (GLP-1) receptor agonists, and dipeptidyl peptidase-IV (DPP-4) inhibitors have been introduced, and use of TZDs has declined due to concerns about congestive heart failure and increased bone fractures. DPP-4 inhibitors and GLP-1 agonists have largely replaced TZDs and compete with SUs as first choice to be added to metformin; they are also used as triple therapy added to metformin and SUs. Increasingly, patients failing to maintain glycemic control with metformin/SU, metformin/DPP-4 inhibitor or metformin/GLP-1 receptor agonist are progressing directly to insulin without having first attempted noninsulin triple therapy. This is largely driven by the convenience and lower hypoglycemic potential of long-acting basal insulin. There is controversy regarding which, if any, prior OADs should be discontinued before initiating insulin therapy, ${ }^{5,6}$ although it is probably most common to discontinue SUs and to maintain metformin $^{7-9}$ in order to reduce hypoglycemic potential and weight gain, while taking advantage of metformin's insulinsensitizing actions.

Vildagliptin is a potent and selective inhibitor of DPP-4 that improves glycemic control in patients with T2DM by blocking the degradation of the incretin hormones, GLP-1 and glucose-dependent insulinotropic polypeptide (GIP). Thus vildagliptin increases plasma levels of the intact biologically active form of both of these peptides ${ }^{10-12}$ and thereby increases the sensitivity and responsiveness of pancreatic $\alpha$-cells and $\beta$-cells to glucose. ${ }^{12}$ Owing to the perception that incretinbased therapies act primarily to reduce postprandial glucose (PPG) rather than fasting plasma glucose (FPG) levels and knowledge that excessive PPG is the predominant dysglycemia early in the T2DM disease process, ${ }^{13}$ it was often recommended that DPP-4 inhibitors be employed early in patients with relatively low A1C (6.5\%-7.5\%) or as an add-on to metformin or a TZD with increasing A1C levels. ${ }^{14}$ However, it is now clear that vildagliptin's effect on FPG is not only secondary to its effect on PPG but also due to a direct effect on overnight hepatic glucose production (HGP) as a result of a long-standing inhibition of DPP-4, covering also the overnight period. ${ }^{12}$ Moreover, there is now increasing evidence that DPP-4 inhibitors, such as vildagliptin, are useful at later stages of T2DM. ${ }^{15,16}$ Furthermore, it was recently demonstrated that vildagliptin's efficacy is independent of disease duration and degree of insulin resistance. ${ }^{17}$
There is a strong rationale for using incretin-based therapies with insulin. In particular, hypoglycemia is the limiting factor in glycemic management for insulin-treated patients with $\mathrm{T} 2 \mathrm{DM},{ }^{18}$ and incretin-based therapies have a low hypoglycemic potential. In fact, there is good scientific basis to expect DPP-4 inhibitors to prevent or counteract hypoglycemia through increased levels of intact (active) GIP $^{10-12}$ that stimulate glucagon secretion under hypoglycemic conditions. ${ }^{19}$ In addition, incretin-based therapy is not associated with weight gain, which is commonly seen with other agents.

In this review we will describe the studies in which vildagliptin has been used in combination with insulin, provide a mechanistic basis to explain the results from these studies, discuss the efficacy and differences in hypoglycemia between the studies, and attempt a consolidated therapeutic recommendation.

\section{Experience with vildagliptin in combination with insulin}

Data regarding vildagliptin in combination with insulin are available from several studies across a time period of more than 7 years. The first study with vildagliptin in combination with insulin by Fonseca et al was completed in $2005 .{ }^{20}$ This was a 24-week, multicenter, randomized, double-blind, placebo-controlled study in which patients with T2DM and inadequate glycemic control (mean baseline A1C 7.5\%$11.0 \%)$ received vildagliptin $(\mathrm{n}=144,50 \mathrm{mg}$ twice daily [bid]) or placebo $(n=152)$ while continuing prior insulin monotherapy. ${ }^{20}$ Randomized patients had advanced disease (mean T2DM duration $\sim 15$ years) and a long history ( $>6$ years) of insulin treatment. The mean daily insulin dose at baseline in the study was $82 \mathrm{U}$, administered on average by three injections per day. Reflecting clinical practice at the time, there was significant short-acting insulin use by almost half of the patients enrolled in the study.

After 24 weeks of treatment, A1C decreased by $0.5 \%$ from a baseline of $8.4 \%(68 \mathrm{mmol} / \mathrm{mol})$ in patients receiving vildagliptin (between-group difference $=-0.3 \%, P=0.010$ ), with only a minimal increase in insulin dose $(\Delta=+1.2 \mathrm{U} /$ day with vildagliptin, $+4.1 \mathrm{U} /$ day with placebo) despite dose adjustments being allowed. Body weight increased slightly in both groups ( $1.3 \mathrm{vs} 0.6 \mathrm{~kg}$ from a baseline of $95 \mathrm{~kg}$ ) with no significant between-group difference. Interestingly, hypoglycemic episodes were reported with a lower frequency and severity with vildagliptin compared to placebo. In the vildagliptin group, only 33 patients experienced a total of 113 events, whereas in the placebo group 45 patients 
experienced a total of 185 events (1.95 vs 2.96 events/ patient year, respectively, $P<0.001)$. In addition, there were six severe (requiring assistance of another person to treat) episodes in the placebo group and none in vildagliptintreated patients $(P=0.032)$. The rate of hypoglycemia was also numerically (albeit not statistically significantly) lower with vildagliptin than placebo in the subgroup of elderly patients $\geq 65$ years (mean age $=71$ years), a patient population particularly susceptible to hypoglycemia due to age-associated impairment in counterregulatory responses. In a double-blind, 28-week extension (overall treatment period 1 year), the hypoglycemia benefit was still evident and the improvement in glycemic control was sustained in the patients continuing to receive vildagliptin $50 \mathrm{mg}$ bid ( $\Delta$ from week $0=-0.5 \%, P<0.001, \mathrm{n}=96) .{ }^{21}$

Limitations of the above study relative to the present clinical practice of insulin therapy include the high mean daily dose of insulin that suppresses endogenous insulin secretion, the significant use of short-acting insulin, as well as the absence of metformin, ie, the exclusive use of insulin monotherapy. A further study evaluating vildagliptin in combination with insulin, intended to be more reflective of the current clinical use of insulin therapy, was therefore performed in 2011. This was a 24-week, multicenter, randomized, double-blind study comparing the effects of vildagliptin ( $50 \mathrm{mg}$ bid, $\mathrm{n}=228$ ) with placebo $(\mathrm{n}=221)$ added to ongoing insulin therapy, with or without metformin. ${ }^{22}$ While the mean T2DM duration (13 years) and mean duration of insulin use ( $\sim 4.5$ years) in this study were similar to the previous study, the mean total daily dose of insulin was about half (41 U/day). In addition, approximately $40 \%$ of patients were receiving a basal insulin regimen, and the remaining about $60 \%$ were treated with premixed insulin. As per the study design, the insulin dose was kept essentially stable over the course of the study ( $\Delta=-1.1$ vs $-0.2 \mathrm{U} /$ day with vildagliptin and placebo, respectively). Consistent with current clinical practice, over $60 \%$ of the patients were also receiving metformin (mean dose $>1900 \mathrm{mg} /$ day) in addition to their insulin regimen.

After 24 weeks of treatment, vildagliptin reduced A1C by $0.8 \%$ (baseline $8.8 \% ; 73 \mathrm{mmol} / \mathrm{mol}$ ), with a between-group difference of $-0.7 \%(P<0.001)$ (Figure 1$)$. Significant reductions of similar magnitude were seen both in the presence and absence of metformin. The markedly lower glucose level achieved with vildagliptin vs placebo added to insulin was not associated with an increased risk of hypoglycemia. A low and comparable percentage of patients in the vildagliptin and placebo treatment groups experienced hypoglycemic events $(8.4 \%$ vs $7.2 \% ; P=$ not significant [NS]), with severe hypoglycemic episodes being experienced by $0.9 \%$ of patients in each treatment group. Treatment was also not associated with weight gain ( $\Delta$ body weight $=+0.1 \mathrm{~kg}$ with vildagliptin vs $-0.4 \mathrm{~kg}$ in the placebo group).

Patients with long-standing T2DM and severe renal impairment (RI) often require insulin therapy. Hyperglycemia in these patients is particularly difficult to manage due to limited therapeutic options as well as frequent comorbidities. In a recent 24 -week study of vildagliptin (50 $\mathrm{mg}$ once daily [qd]) in 515 patients with moderate or severe RI, ${ }^{23}$ the vast majority of patients with severe RI $(\sim 80 \% ; \mathrm{n}=178$; estimated glomerular filtration rate $[\mathrm{eGFR}] \sim 21 \mathrm{~mL} / \mathrm{min} / 1.73 \mathrm{~m}^{2}$ ) were receiving insulin background therapy, which allowed a subanalysis to be performed to evaluate vildagliptin in combination with insulin in this difficult to treat patient population. ${ }^{24}$ Patients had a mean duration of T2DM of $\sim 19$ years and had received insulin therapy for an average of $\sim 5$ years. The mean insulin dose at baseline was $52 \mathrm{U} /$ day (not changing meaningfully in either group during the study), and approximately $85 \%$ of patients received insulin monotherapy, with the majority receiving basal insulin either alone or in a basal/bolus regimen. From a lower baseline A1C $(7.7 \% ; 61 \mathrm{mmol} / \mathrm{mol})$ than in the previous two studies, a marked reduction $(\Delta=-0.9 \%)$ was seen with vildagliptin (between-treatment difference $=-0.6 \%, P<0.001$ ). Despite the mean $\mathrm{A} 1 \mathrm{C}$ after 24 weeks of treatment with vildagliptin being below $7 \%$, where hypoglycemia becomes increasingly more frequent with conventional intensification of treatment, the two treatment groups had an overall comparable hypoglycemia profile. In the vildagliptin group, 19/100 patients $(19.0 \%)$ experienced a total of 36 events, while in the placebo group $11 / 78$ patients $(14.1 \%)$ had a total of 36 events, and there was a similarly low risk of severe hypoglycemia ( $2.0 \%$ with vildagliptin vs $2.6 \%$ with placebo). Body weight did not change in patients receiving vildagliptin $(\Delta=-0.1 \mathrm{~kg})$ and increased slightly (by less than $1 \mathrm{~kg}$ ) in patients receiving placebo.

In all three studies, vildagliptin in combination with insulin showed an overall safety profile consistent with that reported previously from a large pooled safety database..$^{25,26}$

It is further worth mentioning that in addition to the experience described above, there has also been a recent report from an open-label, prospective, nonrandomized, 3-month observational study examining effects of adding vildagliptin or fixed dose vildagliptin/metformin in 300 patients with T2DM inadequately controlled on their 
A

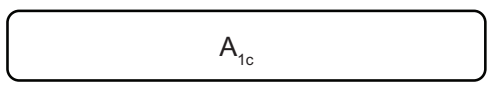

B

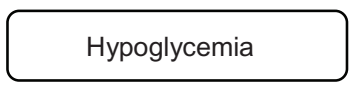

C




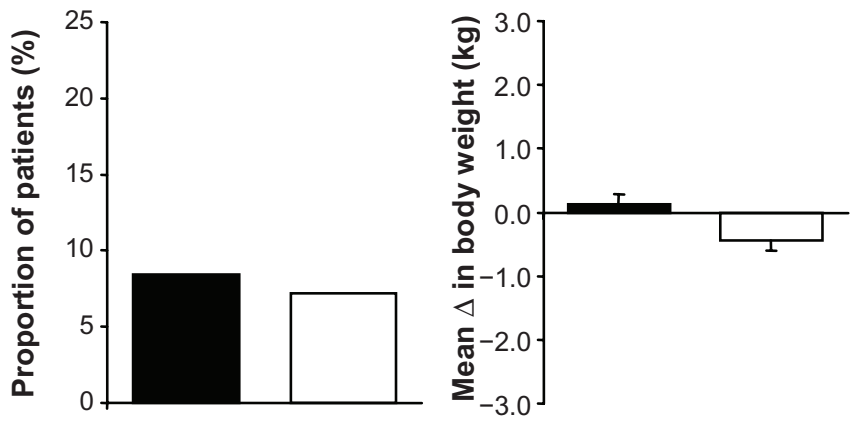

Figure I (A) Adjusted mean ( \pm standard error of the mean) change from baseline to 24-week endpoint in AIC in patients with T2DM adding vildagliptin 50 mg bid (closed bars; $n=221$ ) or placebo (open bars; $n=215$ ) to their ongoing insulin regimen (with or without metformin) and between-group difference (open bars). ap $<0.001$. (B) Proportion of patients with T2DM experiencing confirmed hypoglycemic episodes during 24-week treatment with vildagliptin (50 mg bid, closed bars; $\mathrm{n}=227$ ) or placebo (open bars; $n=221$ ) added to their ongoing insulin regimen (with or without metformin). (C) Mean change from baseline to 24-week endpoint in body weight in patients with T2DM adding vildagliptin $50 \mathrm{mg}$ bid (closed bars; $\mathrm{n}=222$ ) or placebo (open bars; $\mathrm{n}=215$ ) to their ongoing insulin regimen (with or without metformin). ${ }^{22}$ Abbreviations: T2DM, type 2 diabetes; bid, twice daily.

current insulin therapy. ${ }^{27}$ In this real-life setting in India, it was observed that vildagliptin or vildagliptin/metformin fixed dose combination (titrated upward toward targeted glycemic levels) decreased A1C from $9.0 \%$ (75 mmol $/ \mathrm{mol})$ to $7.7 \%$ (61 mmol $/ \mathrm{mol})(P<0.0001)$, with concomitant reduction in the mean daily insulin dose from 36 to $27 \mathrm{U} /$ day. Body weight decreased modestly but significantly (by $\sim 1 \mathrm{~kg}$ ). This report contained no information about hypoglycemia.

Taken together, in a wide variety of patients in the different clinical studies, vildagliptin in combination with insulin improved glycemic control without increased risk of hypoglycemia. The question of the potential mechanistic basis for this benefit therefore arises, which is discussed in the following section.

\section{Mechanistic considerations}

Under hyperglycemic conditions, insulin secretion is high and glucagon secretion is low, resulting in suppression of HGP. Insulin secretion is further augmented if the glucose levels are very high, leading to increased glucose utilization in the muscle. ${ }^{28,29}$ When glucose levels decrease from hyperglycemic levels, the first line of defense against hypoglycemia is the reduction in the insulin secretion rate. This reverses any increase in glucose utilization in muscle and decreases the insulin-glucagon ratio in the liver, leading to increased HGP. If this reduction in insulin secretion fails to prevent glucose levels from falling below $\sim 4.5 \mathrm{mM}$, the second line of defense against hypoglycemia is increased glucagon secretion, further decreasing the insulin-glucagon ratio in the liver and leading to even higher rates of HGP. If glucose levels drop below $\sim 3.5 \mathrm{mM}$, the third line of defense against hypoglycemia is increased catecholamine and cortisol secretion, which in turn further increase the rate of $\mathrm{HGP}^{30,31}$

The incretin hormones GLP-1 and GIP increase the sensitivity of the $\beta$-cells to glucose, leading to a more rapid and pronounced increase in insulin secretion in response to increased glucose levels and a more rapid and pronounced decrease in insulin secretion in response to a decrease in glucose levels. ${ }^{19,32}$ GLP-1 and GIP also increase the sensitivity of the $\alpha$-cells to glucose, leading to a more rapid and pronounced decrease in glucagon secretion in response to increased glucose levels in the hyperglycemic range (GLP-1) and a more rapid and pronounced increase in glucagon secretion in response to a decrease in glucose levels in the hypoglycemic range (GIP). ${ }^{19,33}$ The glucagon responses to hyper- and hypoglycemia were illustrated in a placebo-controlled cross-over study with vildagliptin, which used standard breakfast meal tests followed by hyperinsulinemic-stepped glucose clamps $(7.5,5.0$, and $2.5 \mathrm{mmol} / \mathrm{L}$ glucose).$^{34}$ In that study it was shown that while vildagliptin suppressed inappropriate glucagon secretion during the standard meal tests (by 41\%), it enhanced the glucagon response to hypoglycemia (at the $2.5 \mathrm{mM}$ 
glucose level by 38\%) (Figure 2). Vildagliptin prevents the inactivation of both GLP-1 and GIP. Presumably, GLP-1 decreases glucagon secretion during hyperglycemia, and GIP stimulates glucagon during hypoglycemia.

These glucose-dependent effects of GLP-1 and GIP underlie the glucose-lowering efficacy of DPP-4 inhibitors in patients with T2DM as well as their low propensity to elicit hypoglycemia. Insulin therapy is associated with increased hypoglycemia, and hypoglycemia limits insulin's ability to bring patients to the glycemic goal; the closer insulin brings a patient to the goal, the higher the hypoglycemic rate. Adding vildagliptin to patients on insulin therapy has been shown to lower the glucose levels further - likely via a GLP-1-induced reduction in glucagon during meals - and protected against hypoglycemia - likely via a GIP-induced increase in glucagon levels whenever glucose levels fall into the hypoglycemic range. In order for a GIP-induced increase in glucagon to translate into protection against hypoglycemia, these effects would need to be present during inter-meal and overnight periods when hypoglycemia is likely to occur. Vildagliptin inhibits DPP-4 and thereby prolongs the elevation in plasma levels of the intact and biologically active forms of GLP-1 and GIP ${ }^{12}$ secreted at the beginning of each meal. The nature of vildagliptin's interaction with the DPP-4 enzyme as a substrate (substrate-like enzyme blocker) and its twice daily dosage regimen essentially results in a chronic blocking of DPP-4 inactivation of GLP-1 and GIP, leading to an extension of the physiological increases in GLP-1 and GIP at the beginning of meals, throughout the meals, between meals, and overnight. ${ }^{12}$ In fact, fasting levels of GLP-1 and GIP are elevated in patients treated with vildagliptin. ${ }^{12}$

Besides vildagliptin's effect to enhance glucagon counterregulation via increased glucose sensitivity in the $\alpha$-cells, counterregulation is also enhanced during hypoglycemia by a further reduction in insulin secretion, by diminishing the suppression of glucagon secretion via the paracrine effect of insulin, and by stimulating glucagon secretion via activation of the autonomic nervous system. In the above-mentioned study on glucagon counterregulation during hypoglycemia, vildagliptin treatment enhanced the hypoglycemia-induced inhibition of insulin secretion and there was a clear trend by vildagliptin to increase the activity of the autonomic nervous system as indirectly observed by measuring the marker pancreatic polypeptide. ${ }^{34}$ Therefore, several mechanisms may explain the protective effect of vildagliptin on glucagon counterregulation.

It should also be emphasized that although insulin inhibits glucagon secretion, it is unlikely that peripheral administration of insulin in a therapeutic setting results in sufficient intra-islet insulin levels to elicit such an effect. Therefore, the inappropriate hyperglucagonemia in T2DM remains to be targeted in patients on insulin, and adding a DPP-4 inhibitor will result in this lowering of glucagon.

Vildagliptin has also been studied in patients with type 1 diabetes (with no endogenous insulin secretion). In these patients vildagliptin showed reduced glucagon secretion during meals, leading to better glycemic control. ${ }^{12,35}$ However, in patients with T2DM on basal insulin regimens,
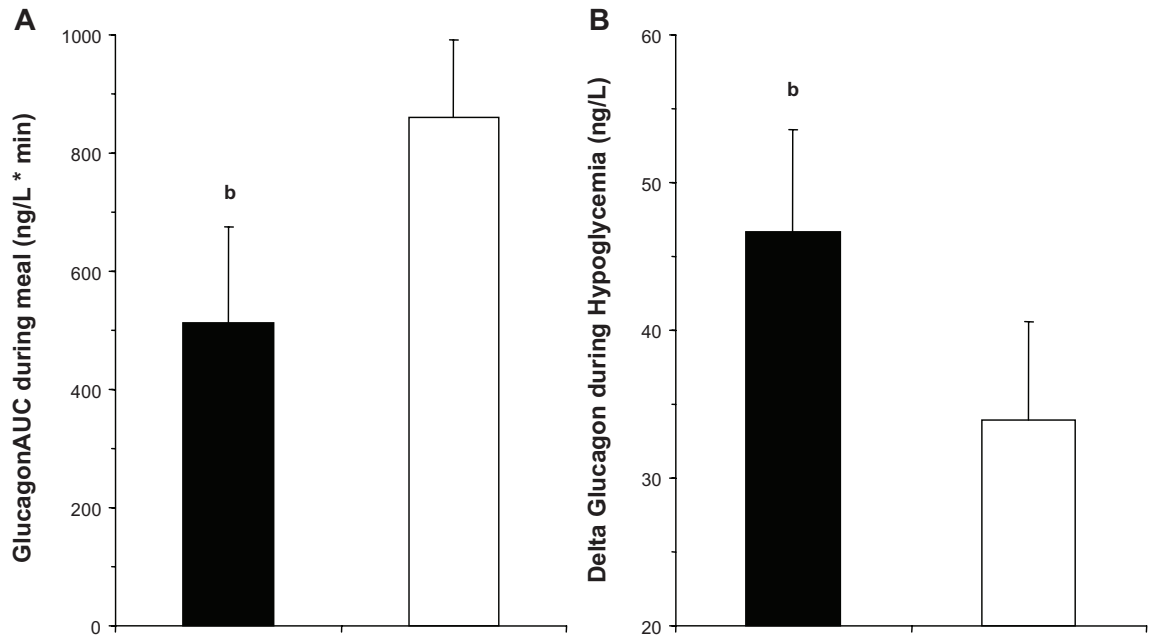

Figure 2 (A) AUC during the first 60 minutes of a standardized mixed meal in drug-naïve patients with T2DM on day 28 of treatment with vildagliptin (I00 mg qd, closed bars) or placebo (open bars) during a crossover study $\left(n=25\right.$ patients). ${ }^{b} P<0.05$. (B) Change in plasma glucagon levels from beginning to end of the $2.5 \mathrm{mM}$ glucose step of a stepped hyperinsulinemic hypoglycemic clamp performed in drug-naive patients with T2DM on day 28 of treatment with vildagliptin (I00 mg qd, closed bars) or placebo (open bars) during a crossover study $\left(\mathrm{n}=25\right.$ patients). ${ }^{34}$

Note: ${ }^{b} P<0.05$.

Abbreviations: AUC, area under the curve; T2DM, type 2 diabetes; qd, once daily. 
there was additionally improved $\beta$-cell function as assessed by the insulin secretion rate relative to glucose during an oral glucose tolerance test. ${ }^{22}$

\section{Therapeutic considerations}

As detailed earlier, all studies conducted with vildagliptin in combination with insulin showed an improvement in glycemic control with no clinically relevant increase in the risk of hypoglycemia. However, there were differences between the studies, both in the degree of the glucoselowering effect of vildagliptin/insulin as well as regarding whether a comparable hypoglycemia profile was seen versus placebo/insulin or even a reduction of hypoglycemia; these merit further discussion/reconciliation.

With respect to efficacy, in the first study by Fonseca et $\mathrm{al}^{20}$ the addition of vildagliptin to insulin monotherapy produced a modest improvement in glycemic control $[-0.5 \%$ from a baseline of $8.4 \%$ and $-0.3 \%$ relative to placebo]. In contrast, in the second study ${ }^{22}$ as well as in the subgroup analysis in patients with severe $\mathrm{RI},{ }^{24}$ robust reductions in A1C $(-0.8 \%$ from a baseline of $8.8 \%$ [ $-0.7 \%$ relative to placebo $]$ and $-0.9 \%$ from a baseline of $7.7 \%[-0.6 \%$ relative to placebo]) were observed. A likely explanation for this difference is that in the first study by Fonseca et al, ${ }^{20}$ patients were chronically exposed to high insulin levels of exogenous origin (mean $>80 \mathrm{U} /$ day), suppressing endogenous insulin secretion, thus an improvement in $\beta$-cell function, a main mechanism of vildagliptin to improve glycemic control, is unlikely to have made a meaningful contribution to the glucose-lowering effect. Thus, the observed efficacy is mainly driven by the contribution that enhanced $\alpha$-cell function can make to improved glycemia. On the other hand, in the second study in patients treated with basal or premixed insulin at a mean dose of $\sim 40 \mathrm{U} /$ day, a significant improvement in $\beta$-cell function was demonstrated with vildagliptin, suggesting that the reduction in $\mathrm{A} 1 \mathrm{C}$ was at least partly due to enhanced $\beta$-cell secretion of insulin. In addition, considering the mode of action of vildagliptin, while not directly assessed in the study, improved $\alpha$-cell function, ie, reduced elevated postprandial and nighttime glucagon levels, can be predicted to have contributed to the $\mathrm{A} 1 \mathrm{C}$ reduction in parallel with the improved $\beta$-cell function, thus yielding the full glucoselowering potential. Similar considerations likely also apply to the subgroup analysis in patients with severe RI, the majority of which were treated with basal insulin either alone or in a basal/bolus regimen at a mean dose of $\sim 50 \mathrm{U} /$ day.

With respect to hypoglycemia, in the first study by Fonseca et $\mathrm{al}^{20}$, the addition of vildagliptin to insulin monotherapy significantly decreased both the frequency and severity of hypoglycemia relative to placebo plus insulin. In the second study as well as in the subgroup analysis in patients with severe RI, vildagliptin added to basal or premixed insulin showed a hypoglycemia profile similar to that of placebo/insulin, ie, the improvement in glycemic control was not associated with an increase in the incidence or severity of hypoglycemic episodes, although the A1C levels were considerably lower in the group treated with vildagliptin. As discussed in detail earlier, this protection against hypoglycemia is likely explained by an enhanced counterregulatory response to incipient hypoglycemia with vildagliptin, bearing in mind that this counterregulation is greatly impaired in insulin-dependent patients with T2DM who have more progressive damage of pancreatic islets compared to patients with less advanced T2DM and treated with OADs. ${ }^{36}$ An open question remains, why, beyond preventing an increase in hypoglycemia as in the other two investigations, an actual reduction in the incidence and severity of hypoglycemia was seen in the Fonseca et al study. ${ }^{20}$ This could potentially be related to the fact that the insulin regimen used in the Fonseca et al study ${ }^{20}$ with high doses and often multiple daily injections of short-acting insulin(s) can cause more and lower excursions into the hypoglycemia range than is expected from the more predominant use of basal insulin and lower doses in the later two studies. For example, the high insulin doses during meals are predicted to increase the rate of post-meal hypoglycemia. Consistent with this thinking, a considerably higher underlying incidence of overall hypoglycemia as well as severe hypoglycemia was seen in the Fonseca et al study ${ }^{20}$ than in the second more recent study ( $29.6 \%$ vs $7.2 \%$ ), respectively, with three times more severe events. As a consequence, once the underlying incidence and/or severity are high enough, one would expect more opportunities to reduce hypoglycemia, which could provide an explanation for the observed results.

Taken together, the differences in the efficacy/hypoglycemia data between the initial and the two later studies are likely due to the differences in the insulin regimens utilized, which reflect the changes in the treatment paradigm of insulin therapy in T2DM toward the predominant use of insulin as a basal regimen (with or without metformin) ${ }^{37}$ vs traditional approaches employed in the past to control postprandial glycemia.

This also has implications if one considers situations in which the use of vildagliptin/DPP-4 inhibitors in combination with insulin could be most desirable, ie, which treatment regimens/combinations are expected to provide the greatest benefit. As outlined earlier, it has become increasingly common 
to maintain metformin treatment in combination with insulin treatment. If DPP-4 inhibitors are used in combination with metformin and basal insulin, metformin and basal insulin would lower basal glucose levels and the DPP-4 inhibitor would provide the bolus action to reduce postprandial glucose levels while having an additional small effect to lower fasting levels further. Alternatively, a reduction in the insulin dose requirements could be achieved. In any case such a treatment regimen would optimally utilize the complementary mechanisms of action of the different treatment components, making it easier for physicians to treat patients to target with insulin therapy without an increased risk of hypoglycemia or weight gain. Further studies are therefore warranted to study this particular combination and patient segment.

\section{Conclusion}

Vildagliptin in combination with insulin has been shown to improve glycemic control with hypoglycemia and weight benefits versus what is commonly seen with intensification of insulin therapy, particularly as a patient approaches the goal. The protection against hypoglycemia seen with vildagliptin has a solid mechanistic basis; it is likely mediated via a GIPinduced increase in glucagon levels whenever glucose levels fall into the hypoglycemic range. Vildagliptin treatment extends the physiological increases of the incretin hormones at the beginning of meals, throughout the meals, between meals, and in the overnight period. The data support the use of vildagliptin in combination with insulin in general, and in line with emerging clinical practice, suggest that treating patients with vildagliptin, metformin, and basal insulin could be an attractive therapeutic option.

\section{Acknowledgments}

The authors gratefully acknowledge the editorial support of Beth Dunning Lower, PhD.

\section{Disclosure}

A Schweizer, JE Foley, and W Kothny are employed by Novartis and own shares. B Ahrén has received research support, honoraria for speaking engagements, and served on advisory boards for Novartis.

\section{References}

1. UK Prospective Diabetes Study Group. Intensive blood-glucose control with sulphonylureas or insulin compared with conventional treatment and risk of complications in patients with type 2 diabetes (UKPDS 33). UK Prospective Diabetes Study (UKPDS) Group. Lancet. 1998;352:837-853.

2. Colwell JA. Intensive insulin therapy in type II diabetes: rationale and collaborative clinical trial results. Diabetes. 1996;45(Suppl 3): S87-S90.
3. Turner RC, Holman RR. Lessons from UK prospective diabetes study. Diabetes Res Clin Pract. 1995;28(Supp1):S151-S157.

4. Diabetes Control and Complications Trial Research Group. The effect of intensive treatment of diabetes on the development and progression of long-term complications in insulin-dependent diabetes mellitus. N Engl J Med. 1993;329:977-986.

5. Janka HU, Plewe G, Riddle MC, Kliebe-Frisch C, Schweitzer MA, Yki-Jarvinen $\mathrm{H}$. Comparison of basal insulin added to oral agents versus twice-daily premixed insulin as initial insulin therapy for type 2 diabetes. Diabetes Care. 2005;28:254-259.

6. Peters KR. Intensifying treatment of type 2 diabetes mellitus: adding insulin. Pharmacotherapy. 2011;31:54S-64S.

7. Raskin P. Why insulin sensitizers but not secretagogues should be retained when initiating insulin in type 2 diabetes. Diabetes Metab Res Rev. 2008;24:3-13.

8. Yki-Jarvinen H. Combination therapies with insulin in type 2 diabetes. Diabetes Care. 2001;24:758-767.

9. Swinnen SG, Dain MP, Mauricio D, Devries JH, Hoekstra JB, Holleman F Continuation versus discontinuation of insulin secretagogues when initiating insulin in type 2 diabetes. Diabetes Obes Metab. 2010;12: 923-925.

10. HeYL, Serra D, Wang Y et al. Pharmacokinetics and pharmacodynamics of vildagliptin in patients with type 2 diabetes mellitus. Clin Pharmacokinet. 2007;46:577-588.

11. Rosenstock J, Foley JE, Rendell M, et al. Effects of the dipeptidyl peptidase-IV inhibitor vildagliptin on incretin hormones, islet function, and postprandial glycemia in subjects with impaired glucose tolerance. Diabetes Care. 2008;31:30-35.

12. Ahren B, Schweizer A, Dejager S, Villhauer EB, Dunning BE, Foley JE. Mechanisms of Action of the DPP-4 Inhibitor Vildagliptin in Man. Diabetes Obes Metab. 2011;13:775-783.

13. Monnier L, Colette C. Contributions of fasting and postprandial glucose to hemoglobin A1c. Endocr Pract. 2006;12(Suppl 1):42-46.

14. Rodbard HW, Jellinger PS, Bloomgarden ZT. AACE/ACE glycemic control algorithm consensus panel. Endocr Pract. 2009;15: 541-559.

15. Ahluwalia R, Vora J. Emerging role of insulin with incretin therapies for management of type 2 diabetes. Diabetes Ther. 2011;2:146-161.

16. Rizos EC, Ntzani EE, Papanas N, et al. Combination Therapies of DPP4 Inhibitors and GLP1 Analogues with Insulin in Type 2 Diabetic Patients: A Systematic Review. Curr Vasc Pharmacol. Epub 2012 Jun 22.

17. Schweizer A, Dejager S, Foley JE. Impact of insulin resistance, body mass index, disease duration, and duration of metformin use on the efficacy of vildagliptin. Diabetes Ther. 2012;3:8.

18. Cryer PE. Hypoglycaemia: the limiting factor in the glycaemic management of Type I and Type II diabetes. Diabetologia. 2002;45: 937-948.

19. Christensen M, Vedtofte L, Holst JJ, Vilsboll T, Knop FK. Glucosedependent insulinotropic polypeptide: a bifunctional glucose-dependent regulator of glucagon and insulin secretion in humans. Diabetes. 2011;60:3103-3109.

20. Fonseca V, Schweizer A, Albrecht D, Baron MA, Chang I, Dejager S. Addition of vildagliptin to insulin improves glycaemic control in type 2 diabetes. Diabetologia. 2007;50:1148-1155.

21. Fonseca V, Baron M, Shao Q, Dejager S. Sustained efficacy and reduced hypoglycemia during one year of treatment with vildagliptin added to insulin in patients with type 2 diabetes mellitus. Horm Metab Res. 2008;40:427-430

22. Kothny W, Foley J, Kozlovski P, Shao Q, Gallwitz B, Lukashevich V. Improved glycaemic control with vildagliptin added to insulin, with or without metformin, in patients with type 2 diabetes mellitus. Diabetes Obes Metab. 2013;15:252-257.

23. Lukashevich V, Schweizer A, Shao Q, Groop PH, Kothny W. Safety and efficacy of vildagliptin versus placebo in patients with type 2 diabetes and moderate or severe renal impairment: a prospective 24-week randomized placebo-controlled trial. Diabetes Obes Metab. 2011;13:947-954. 
24. Lukashevich V, Schweizer A, Foley JE, Dickinson S, Groop PH, Kothny W. Efficacy of vildagliptin in combination with insulin in patients with type 2 diabetes and severe renal impairment. Vasc Health Risk Manag. 2013;9:21-28.

25. Ligueros-Saylan M, Foley JE, Schweizer A, Couturier A, Kothny W. An assessment of adverse effects of vildagliptin versus comparators on the liver, the pancreas, the immune system, the skin and in patients with impaired renal function from a large pooled database of Phase II and III clinical trials. Diabetes Obes Metab. 2010;12:495-509.

26. Schweizer A, Dejager S, Foley JE, Kothny W. Assessing the general safety and tolerability of vildagliptin: value of pooled analyses from a large safety database versus evaluation of individual studies. Vasc Health Risk Manag. 2011;7:49-57.

27. Ved P, Shah S. Evaluation of vildagliptin and fixed dose combination of vildagliptin and metformin on glycemic control and insulin dose over 3 months in patients with type 2 diabetes mellitus. Indian J Endocrinol Metab. 2012;16(Suppl 1):S110-S113.

28. Bertoldo A, Pencek RR, Azuma K, et al. Interactions between delivery, transport, and phosphorylation of glucose in governing uptake into human skeletal muscle. Diabetes. 2006;55:3028-3037.

29. Pencek RR, Bertoldo A, Price J, Kelley C, Cobelli C, Kelley DE. Doseresponsive insulin regulation of glucose transport in human skeletal muscle. Am J Physiol Endocrinol Metab. 2006;290:E1124-E1130.
30. Cryer PE. Hypoglycemia: still the limiting factor in the glycemic management of diabetes. Endocr Pract. 2008;14:750-756.

31. Cryer PE. The barrier of hypoglycemia in diabetes. Diabetes. 2008;57:3169-3176.

32. Ahren B, Holst JJ, Mari A. Characterization of GLP-1 effects on betacell function after meal ingestion in humans. Diabetes Care. 2003;26: $2860-2864$

33. Holst JJ, Christensen M, Lund A, et al. Regulation of glucagon secretion by incretins. Diabetes Obes Metab. 2011;13(Suppl 1):89-94.

34. Ahren B, Schweizer A, Dejager S, et al. Vildagliptin enhances islet responsiveness to both hyper- and hypoglycemia in patients with type 2 diabetes. J Clin Endocrinol Metab. 2009;94:1236-1243.

35. Foley JE, Ligueros-Saylan M, He YL, et al. Effect of vildagliptin on glucagon concentration during meals in patients with type 1 diabetes. Horm Metab Res. 2008;40:727-730.

36. Ferrannini E, Groop PH, Nauck M, Fioretto P, Hach T, Thomas M. The Significance of the Kidney in Diabetes. Konigswinter, Germany: infill Kommunikation $\mathrm{GmbH} ; 2012$.

37. Inzucchi SE, Bergenstal RM, Buse JB, et al. Management of hyperglycaemia in type 2 diabetes: a patient-centered approach. Position statement of the American Diabetes Association (ADA) and the European Association for the Study of Diabetes (EASD). Diabetologia. 2012;55:1577-1596.
Vascular Health and Risk Management

\section{Publish your work in this journal}

Vascular Health and Risk Management is an international, peerreviewed journal of therapeutics and risk management, focusing on concise rapid reporting of clinical studies on the processes involved in the maintenance of vascular health; the monitoring, prevention and treatment of vascular disease and its sequelae; and the involvement of

\section{Dovepress}

metabolic disorders, particularly diabetes. This journal is indexed on PubMed Central and MedLine. The manuscript management system is completely online and includes a very quick and fair peer-review system, which is all easy to use. Visit http://www.dovepress.com/ testimonials.php to read real quotes from published authors. 